\title{
Infrared study of the southern Galactic star-forming region associated with IRAS 14416-5937 ${ }^{\star}$
}

\begin{abstract}
S. Vig ${ }^{\star}$, S. K. Ghosh, D. K. Ojha, and R. P. Verma
Tata Institute of Fundamental Research, Mumbai 400 005, India

e-mail: sarita@arcetri.astro.it

Received 31 May 2006 / Accepted 26 September 2006

ABSTRACT

Aims. Our goal was to carry out an infrared study of the southern Galactic massive star-forming region associated with IRAS 14416-5937.

Methods. This star-forming region has been mapped simultaneously in two far infrared bands at $\sim 150$ and $210 \mu \mathrm{m}$, using the TIFR 1 -m balloon borne telescope with $\sim 1^{\prime}$ angular resolution. We have used 2MASS $J H K_{\mathrm{s}}$, as well as Spitzer-GLIMPSE data of this region to study the stellar populations of the embedded young cluster. This region comprises two sources, designated as A and B and separated by $\sim 2 \mathrm{pc}$. The spectrum of a region located close to the source A obtained using the Long Wavelength Spectrometer (LWS) on board the Infrared Space Observatory (ISO) is presented. Emission from warm dust and from Unidentified Infrared Bands (UIBs) is estimated using the mid-infrared data of the MSX survey.

Results. The spatial distributions of (1) the temperature of cool dust and (2) optical depth at $200 \mu \mathrm{m}$ have been obtained taking advantage of the similar beams in both the TIFR bands. A number of atomic fine structure lines have been detected in the ISO-LWS spectrum, which have been used to estimate the electron density and the effective temperature of the ionising radiation in this region. From the near- and mid-infrared images, we identify a dust lane due north-west of source A. The dust lane is populated by Class I type sources. Class II type sources are found further along the dust lane as well as below it. Self consistent radiative transfer models of the two sources (A and B) are in good agreement with the observed spectral energy distributions.

Conclusions. The spatial distribution of young stellar objects in and around the dust lane suggests that active star formation is taking place along the dust lane and is possibly triggered by the expanding HII regions of A and B.
\end{abstract}

Key words. infrared: ISM - ISM: H II regions - ISM: individual objects: IRAS 14416-5937 - stars: pre-main sequence

\section{Introduction}

The southern Galactic high mass star-forming region associated with IRAS 14416-5937 is located at a distance of $2.8 \mathrm{kpc}$ (Busfield et al. 2006). It corresponds to the radio source G316.8-0.1. A number of masers and molecular lines have been detected close to this star-forming region. Both $\mathrm{OH}$ (Caswell \& Haynes 1987) and $\mathrm{H}_{2} \mathrm{O}$ (Caswell et al. 1989) masers have been observed here. A methanol $\left(\mathrm{CH}_{3} \mathrm{OH}\right)$ maser (Caswell et al. 1995) showing variability has also been detected here. $\mathrm{NH}_{3}$ (Vilas-Boas et al. 2000), CI (Huang et al. 1999), CO (Whiteoak et al. 1982; White \& Phillips 1983), CS (Bronfman et al. 1996; Juvela 1996), and $\mathrm{H}_{2} \mathrm{CO}$ (Gardner \& Whiteoak 1984) lines have been detected in this star-forming region. Walsh et al. (1998) present high angular resolution $\left(\sim 1.5^{\prime \prime}\right)$ radio continuum and methanol maser data at $6.67 \mathrm{GHz}$ for this source as a part of their survey. Given the location of IRAS 14416-5937 in the extreme southern sky, no detailed study of this region exists in the literature. In this Paper, we have carried out a systematic study of the star-forming region associated with IRAS 14416-5937.

IRAS 14416-5937 has been studied in the infrared wavebands with the aim of investigating the emission from dust, the dust temperature, the energetics, and the stellar populations of

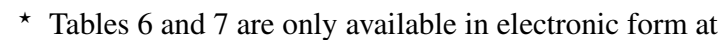
http: //www . aanda.org

$\star \star$ Present address: INAF-Osservatorio Astrofisico di Arcetri, Largo E. Fermi, 550125 Firenze, Italy. the associated cluster in this region. In Sect. 2, we present the far infrared observations and a description of other available data used in this Paper. Section 3 describes the results, and Sect. 4 deals with the radiative transfer modelling carried out. In Sect. 5, we discuss all the results, and a brief summary is presented in Sect. 6.

\section{Observations and data reduction}

\subsection{Far infrared observations}

The Galactic star-forming region associated with IRAS 14416-5937 has been observed using a two-band far infrared (FIR) photometer system at the Cassegrain focus of the TIFR $100 \mathrm{~cm}(f / 8)$ balloon borne telescope. The observations were carried out during the balloon flight from the TIFR Balloon Facility, Hyderabad in India (latitude $17^{\circ} .47$ north, longitude 78.57 east) on Feb. 20, 1994. Details of the telescope and the observational procedure are given by Ghosh et al. (1988). The two FIR bands use a pair of $2 \times 3$ composite silicon bolometer arrays, cooled to $0.3 \mathrm{~K}$ by liquid ${ }^{3} \mathrm{He}$, which view identical parts of the sky simultaneously. The field of view corresponding to each detector is $1.6^{\prime}$. The sky is chopped along the crosselevation axis at $10 \mathrm{~Hz}$ with a throw of $4.2^{\prime}$. The bandpasses of the two bands are defined by sets of cool filters. The spectral response was measured with a Michelson interferometer using a Golay cell as a comparison detector. The effective wavelengths of the two FIR bands are 150 and $210 \mu \mathrm{m}$, respectively. These 
effective wavelengths correspond to a source spectrum of a $30 \mathrm{~K}$ gray body with an emissivity dependence of $\epsilon_{\lambda} \propto \lambda^{-2}$. The absolute positions were calibrated using the observations of catalogued stars with the optical photometer located at the focal plane of the telescope. The field of view of this optical photometer is offset with respect to the infrared field. The planet Jupiter was observed for absolute flux calibration as well as for the determination of the instrumental Point Spread Function (PSF).

The simultaneous mapping in the two FIR bands was carried out by raster-scanning the telescope along the cross-elevation axis across the target area under study and stepping along the elevation at both the ends of the scans. A $\sim 32^{\prime} \times 20^{\prime}$ region centred around IRAS 14416-5937 was mapped. The FIR signals were gridded into a two-dimensional (elevation $\times$ cross-elevation) matrix with a pixel size of $0.3^{\prime} \times 0.3^{\prime}$. The observed chopped signal matrix was deconvolved using the Maximum Entropy Method similar to that of Gull \& Daniell (1978; see Ghosh et al. 1988, for details). An angular resolution of $\sim 1^{\prime}$ has been achieved in the FIR maps using this method. The estimated error on absolute flux densities for the TIFR bands is $\sim 10 \%$, primarily from the calibration uncertainties using the planet.

\subsection{Other available data sets}

\subsubsection{IRAS-HIRES}

The Infrared Astronomical Satellite (IRAS) survey data at all the four wavelength bands $(12,25,60,100 \mu \mathrm{m})$ for the region around IRAS 14416-5937 were HIRES-processed at the Infrared Processing and Analysis Center (IPAC), Caltech. HIRES processing employs the Maximum Correlation Method (MCM, Aumann et al. 1990) to construct (resolution enhanced) co-added images. These maps have been used in the present study to quantify the flux densities and angular sizes at the four infrared bands. They have also been used to generate the temperature and optical depth maps of interstellar dust. An upper limit on error in flux density in each band has been estimated by integrating several circular regions $\left(3^{\prime}\right.$ diameter) with no point-like source, in the local neighbourhood of the respective maps.

\subsubsection{MSX}

The Midcourse Space Experiment (MSX) was a satellite experiment that surveyed the entire Galactic plane within $|\mathrm{b}| \leq 5^{\circ}$ in four mid-infrared wavebands: $8.3,12.1,14.7$, and $21.3 \mu \mathrm{m}$ with a spatial resolution of $\sim 18^{\prime \prime} .3$ (in all four bands) during 1996-1997 (Price et al. 2001). The infrared instrument on MSX, designated SPIRIT III, was a $35 \mathrm{~cm}$ clear aperture off-axis telescope with focal plane arrays. In the present study, we have used the panoramic images of the region around IRAS 144165937 to extract sources and obtain the integrated flux densities for constructing the spectral energy distribution (SED) of IRAS 14416-5937. Upper limits to errors on flux densities have been estimated using a procedure similar to that for IRASHIRES (see Sect. 2.2.1).

\subsection{3. $2 \mathrm{MASS}$}

The Two Micron All Sky Survey (2MASS) used two highlyautomated 1.3-m telescopes, one at Mt. Hopkins, Arizona (USA), and one at Cerro Tololo Inter-American Observatory (CTIO), Chile, to uniformly scan the entire sky in three nearinfrared bands: $J(1.25 \mu \mathrm{m}), H(1.65 \mu \mathrm{m})$, and $K \mathrm{~s}(2.17 \mu \mathrm{m})$, using a pixel size of 2.0". The survey was completed in 2001 . We have used the point sources from the region around IRAS 14416-5937 from the 2MASS Point Source Catalog (PSC) in this study. The 2MASS PSC is complete down to $J \leq 15.8$, $H \leq 15.1$, and $K_{\mathrm{s}} \leq 14.3 \mathrm{mag}$ for $S / N>10$, in the absence of confusion. The $J, H$, and $K_{\mathrm{s}}$ magnitudes of the extracted sources have been used to make colour-magnitude and colour-colour diagrams to study the embedded cluster in this region. The $J H K_{\mathrm{s}}$ magnitudes and images were taken from IPAC.

\subsubsection{Spitzer-GLIMPSE}

The Spitzer Space Telescope (Werner et al. 2004) was launched in space in August 2003 and consists of a 0.85-m telescope with three cryogenically cooled instruments: InfraRed Array Camera (IRAC), InfraRed Spectrograph, and Multiband Imaging Photometer for Spitzer. IRAC is a four-channel camera that provides simultaneous $5.2^{\prime} \times 5.2^{\prime}$ images at 3.6, 4.5, 5.8 , and $8 \mu \mathrm{m}$ with a pixel size of $1.2^{\prime \prime} \times 1.2^{\prime \prime}$ (Fazio et al. 2004). In the GLIMPSE (Galactic Legacy Infrared Midplane Survey Extraordinaire, Benjamin et al. 2003) project, Spitzer Space Telescope surveys approximately 220 square degrees of the Galactic plane covering a latitude range of $|b|<1^{\circ}$ and a longitude range of $10^{\circ} \leq l \leq 65^{\circ},-65^{\circ} \leq l \leq-10^{\circ}$. This survey is carried out in the 4 IRAC bands. The cata$\log$ lists sources within each surveyed $2^{\circ} \times 2^{\circ}$ region. The sources around IRAS 14416-5937 have been extracted from the GLIMPSE More Complete Archive. The GLIMPSE archive catalogs contain point sources with peak signal-to-noise ratio greater than 5 in at least one band. The magnitudes of the extracted sources have been used in making the colour-colour diagram. The Spitzer-GLIMPSE images have been obtained using the software "Leopard". These images have been used to study the spatial distribution of sources as well as mid-infrared emission from this region.

\subsubsection{SUMSS}

The Sydney University Molonglo Sky Survey (SUMSS) is a radio imaging survey of the southern sky $\left(\delta<-30^{\circ}\right.$, Bock et al. 1999). This survey, using the Molonglo Observatory Synthesis Telescope (MOST), is being carried out at $843 \mathrm{MHz}$. The MOST consists of two cylindrical paraboloids, $778 \mathrm{~m} \times 12 \mathrm{~m}$, separated by $15 \mathrm{~m}$ and aligned east-west. The radio image of IRAS 14416-5937, extracted from the SUMSS Archive, has been used to study the distribution of ionised gas around this region. The synthesised beam size is $43^{\prime \prime} \times 50^{\prime \prime}$.

\subsubsection{ISO}

The Infrared Space Observatory (ISO) was an astronomical satellite experiment consisting of a telescope with primary mirror of size $60 \mathrm{~cm}$, operational between 1995 and 1998. The various scientific instruments on board the ISO operated between 2.5-240 $\mu \mathrm{m}$. We have used the data from the Long Wavelength Spectrometer (LWS, Clegg et al. 1996) for a region close to IRAS 14416-5937 between 43-197 $\mu \mathrm{m}$. The LWS spectrum is for a region centred at $\alpha_{2000}=14^{\mathrm{h}} 45^{\mathrm{m}} 21.0^{\mathrm{s}}, \delta_{2000}=$ $-59^{\circ} 48^{\prime} 14^{\prime \prime}$. The version of the ISO data used in this Paper corresponds to the Highly Processed Data Product (HPDP) sets called "Uniformly processed LWS L01 spectra" by Lloyd et al. (2003), obtained from the ISO Data Archive. 

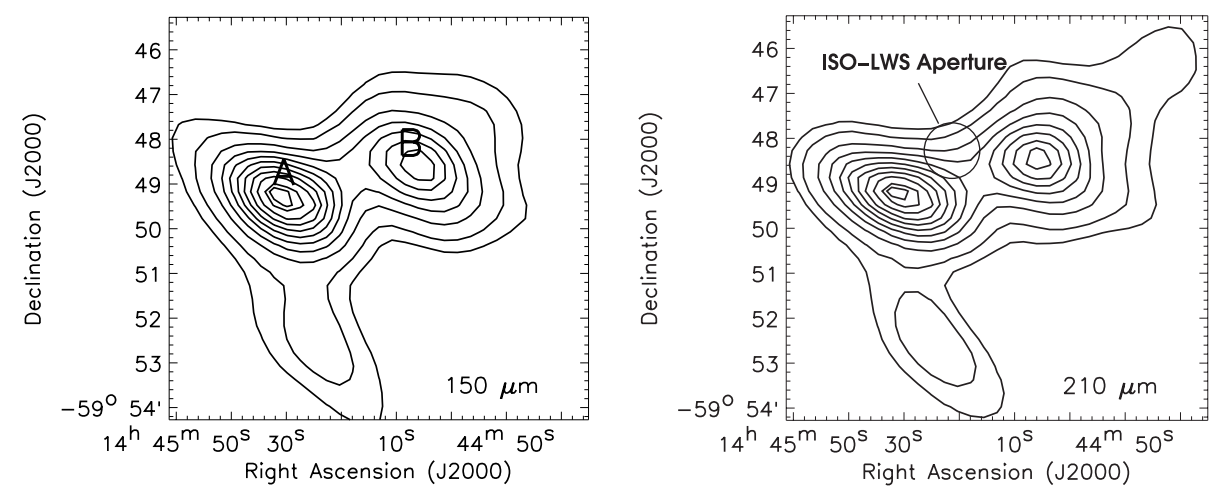

Fig. 1. The intensity map for the region around IRAS 14416-5937 at $150 \mu \mathrm{m}($ left $)$ and $210 \mu \mathrm{m}$ (right). Contour levels are at 5, 10, 20, 30, 40, 50, $60,70,80,90$, and $95 \%$ of peak intensity of $2450 \mathrm{Jy} / \mathrm{sq}$ arcmin (left) and $1367 \mathrm{Jy} / \mathrm{sq}$ arcmin (right).
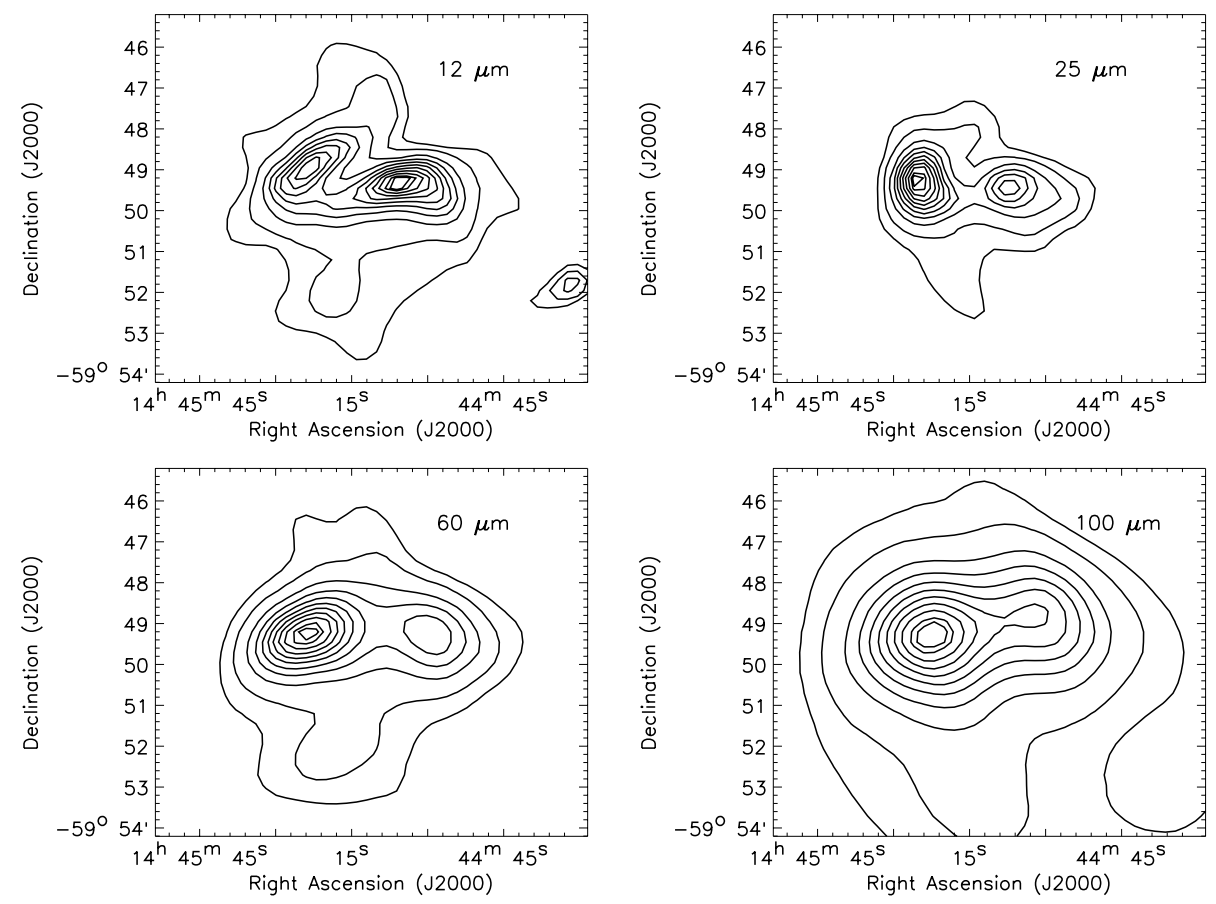

Fig. 2. The IRAS-HIRES intensity map for the region covering IRAS 14416-5937 at $12 \mu \mathrm{m}$ (top left), $25 \mu \mathrm{m}$ (top right), $60 \mu \mathrm{m}$ (bottom left), and $100 \mu \mathrm{m}$ (bottom right). The contours are at 5, 10, 20, 30, 40, 50, 60, 70, 80, 90, and 95\% of the peak value of $155 \mathrm{Jy} \mathrm{arcmin}^{-2}, 1380 \mathrm{Jy} \mathrm{arcmin}^{-2}$,

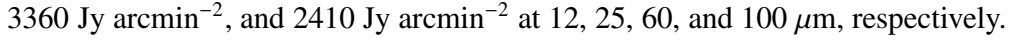

\section{Results}

\subsection{Emission from dust}

The thermal emission from dust in the TIFR-bands at 150 and $210 \mu \mathrm{m}$ is shown in Fig. 1. These deconvolved maps show emission from two sources designated A (east) and B (west). The dynamic range of the maps is quite good and contours are shown up to the 5\% level of the respective peak intensities (2450 Jy/sq arcmin and $1367 \mathrm{Jy} / \mathrm{sq}$ arcmin at 150 and $210 \mu \mathrm{m}$, respectively). The HIRES-processed maps at all the four IRAS bands $(12,25,60$, and $100 \mu \mathrm{m})$ are shown in Fig. 2. Similar to the TIFR maps, both the peaks A and B are seen clearly in the 12,25 , and $60 \mu \mathrm{m}$ maps, but only a hint of B appears at $100 \mu \mathrm{m}$. The peak intensities in the HIRES maps correspond to $155,1380,3360$, and $2410 \mathrm{Jy} / \mathrm{sq}$ arcmin at 12,25 , 60 , and $100 \mu \mathrm{m}$, respectively. The flux densities, obtained by integrating circular regions of diameter $3^{\prime}$ centred on peaks A and $\mathrm{B}$ from the TIFR, IRAS-HIRES, and MSX maps, are listed in Table 1. The IRAS PSC lists a single source in this region.
The corresponding flux densities and position of the IRAS PSC source are also listed in the table.

Although the IRAS-HIRES maps have a much higher dynamic range, the angular resolution of TIFR maps are superior to the IRAS maps (at least at 60 and $100 \mu \mathrm{m}$ ) because of the smaller and circular beams employed. Since the TIFR beams are nearly identical at both the FIR bands and all the observations are simultaneous, this data set is useful in mapping the colour temperature and dust optical depth with good angular resolution. The flux density, $F_{v}$, for optically thin emission can be written as

$F_{v}=\Omega B_{v}\left(T_{\mathrm{d}}\right) \tau_{v}$,

where $\Omega$ is the solid angle of the region under consideration, $B_{v}$ is the Planck function, and $T_{\mathrm{d}}$ is the dust temperature. Assuming $\tau_{v} \propto v^{\beta}$, it can be shown that the ratio of flux densities at any two wavelengths is a function of $T_{\mathrm{d}}$ and $\beta$. For various dust temperatures $\left(T_{\mathrm{d}}\right)$ and an assumed value of $\beta$, a look-up table is generated for the ratio of flux densities. The colour temperature and optical depth maps have been generated using this interpolation table relating the ratio of signals detected for the 
Table 1. Flux density details of IRAS 14416-5937.

\begin{tabular}{|c|c|c|c|c|c|c|c|c|c|c|c|}
\hline \multirow[t]{3}{*}{ Source } & \multirow{3}{*}{$\begin{array}{c}\text { Position } \\
\text { (J2000) }\end{array}$} & \multicolumn{10}{|c|}{ Flux density ${ }^{a}(\mathrm{Jy})$ for $\lambda(\mu \mathrm{m})$} \\
\hline & & \multicolumn{2}{|c|}{ TIFR images } & \multicolumn{4}{|c|}{ IRAS-HIRES images } & \multicolumn{4}{|c|}{ MSX images } \\
\hline & & 210 & 150 & 100 & 60 & 25 & 12 & 21.3 & 14.7 & 12.1 & 8.3 \\
\hline $14416-5937-\mathrm{A}$ & $14^{\mathrm{h}} 45^{\mathrm{m}} 25.4^{\mathrm{s}}-59^{\circ} 49^{\prime} 11^{\prime \prime}$ & 4076 & 7545 & 9442 & 9345 & 2319 & 295 & 1528 & 504 & 334 & 121 \\
\hline $14416-5937-B$ & $14^{\mathrm{h}} 45^{\mathrm{m}} 07.8^{\mathrm{s}}-59^{\circ} 49^{\prime} 00^{\prime \prime}$ & 3451 & 5601 & 6791 & 5750 & 1407 & 321 & 1159 & 468 & 351 & 132 \\
\hline \multicolumn{12}{|c|}{ IRAS PSC } \\
\hline $14416-5937$ & $14^{\mathrm{h}} 45^{\mathrm{m}} 27.9^{\mathrm{s}}-59^{\circ} 49^{\prime} 14^{\prime \prime}$ & & & 16100 & 6840 & 766 & 140 & & & & \\
\hline
\end{tabular}

${ }^{a}$ Fluxes obtained by integrating over a circular region of diameter $3^{\prime}$.
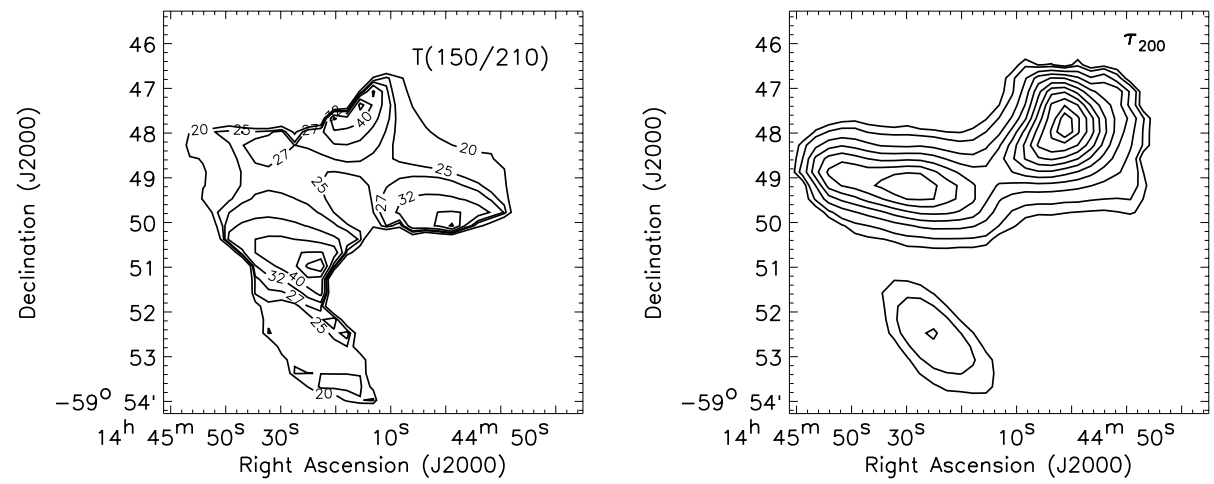

Fig. 3. The distribution of dust temperature $T(150 / 210)$ (left), and optical depth at $200 \mu \mathrm{m}, \tau_{200}$, $($ right $)$ from the region around IRAS $14416-5937$ assuming a dust emissivity law of $\epsilon_{\lambda} \propto \lambda^{-2}$. The isotherms correspond to 20, 25, 27, 32, 40, 50, and $60 \mathrm{~K}$. The $\tau_{200}$ contours represent 5, 10, 20, $30,40,50,60,70,80$, and $90 \%$ of the peak value of 0.06 .

two bands to the dust temperature for the assumed emissivity law, $\epsilon_{\lambda} \propto \lambda^{-2}$. It is important to note that the morphology of the contours of dust temperature and opacity are not sensitive to the assumption $\beta$. Further details can be found in Appendix A of Mookerjea et al. (2000). The generated maps of colour temperature T(150/210) and dust optical depth at $200 \mu \mathrm{m}\left(\tau_{200}\right)$ are shown in Fig. 3.

Using the emission in the MSX bands $(8.3,12.1,14.7$, $21.3 \mu \mathrm{m}$ ) for the region around IRAS 14416-5937, we have modelled the thermal continuum from interstellar dust along with emission in the Unidentified Infrared Bands (UIBs) following the scheme developed by Ghosh \& Ojha (2002). In this scheme, the emission from each pixel in the MSX images is a combination of two components: (i) thermal continuum from dust grains (gray body) and (ii) the emission from the UIB features falling within the MSX band. The scheme assumes that dust emissivity follows the power law of the form $\epsilon_{\lambda} \propto \lambda^{-1}$ and the total radiance due to UIBs in the $12 \mu \mathrm{m}$ band is proportional to that in the $8 \mu \mathrm{m}$ band. The dust emissivity law depends on wavelength. We have used emissivity laws, $\epsilon_{\lambda} \propto \lambda^{-1}$, for $\lambda<100 \mu \mathrm{m}$, and $\epsilon_{\lambda} \propto \lambda^{-2}$, for $\lambda>100 \mu \mathrm{m}$, which is generally used (Scoville \& Kwan 1976). A self consistent non-linear chi-square minimisation technique is used to estimate the total emission from the UIBs, dust temperature, and optical depth in the mid-infrared $(10 \mu \mathrm{m})$. The spatial distribution of UIB emission is shown in Fig. 4. The peak strength of the modelled UIB emission is $1.3 \times 10^{-4} \mathrm{~W} \mathrm{~m}^{-2} \mathrm{Sr}^{-1}$, close to peak $\mathrm{A}$.

\subsection{Emission from gas}

The radio continuum emission from the region around IRAS 14416-5937 from SUMSS survey at $843 \mathrm{MHz}$ is shown in Fig. 5. The rms noise in the map is $\sim 6 \mathrm{mJy} / \mathrm{beam}$. The

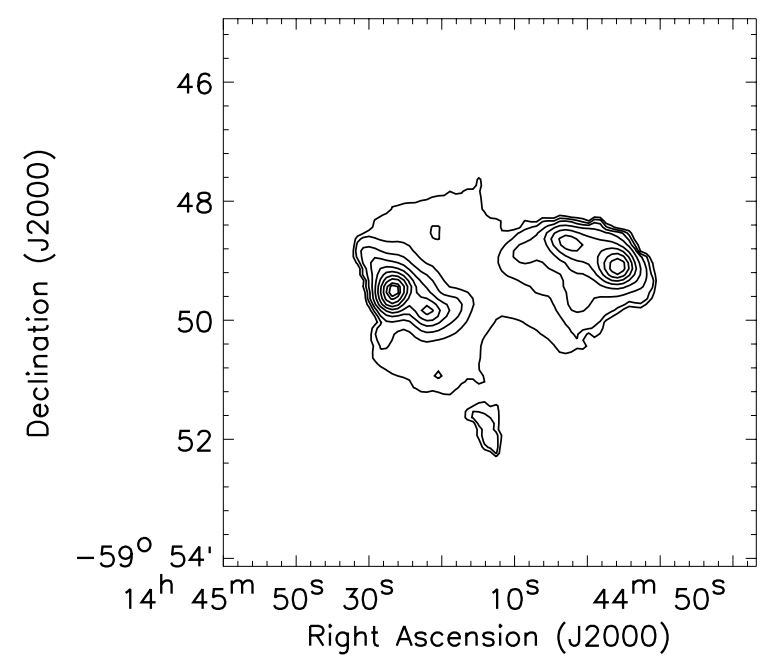

Fig. 4. The emission in UIBs for the region around IRAS 14416-5937. The contour levels are at $1,5,10,20,30,40,50,60,65,70,80,90$, and $95 \%$ of peak value of $1.3 \times 10^{-4} \mathrm{~W} \mathrm{~m}^{-2} \mathrm{Sr}^{-1}$.

radio emission peaks at $\left(\alpha_{2000}=14^{\mathrm{h}} 45^{\mathrm{m}} 23.52^{\mathrm{s}}, \delta_{2000}=\right.$ $\left.-59^{\circ} 49^{\prime} 25.0^{\prime \prime}\right)$. The integrated radio flux density up to $5 \%$ contour level is $\sim 37.5 \mathrm{Jy}$ over $30 \mathrm{arcmin}^{2}$.

The ISO-LWS beam, centred at a location that is $\sim 1.4^{\prime}$ to the north-west of IRAS 14416-5937 - A is shown in Fig. 1 (right). This spectrum, extending from $43-197 \mu \mathrm{m}$ is shown in Fig. 6. A number of lines are prominently detected. The atomic fine structure lines with good signal-to-noise ratios are identified in the figure, and their line fluxes are given in Table 2. The spectrum is dominated by the fine structure lines of [N II], [N III], [O III], [C II], and [OI]. The line fluxes are extracted by fitting Gaussian functions and integrating the area under the curve after 


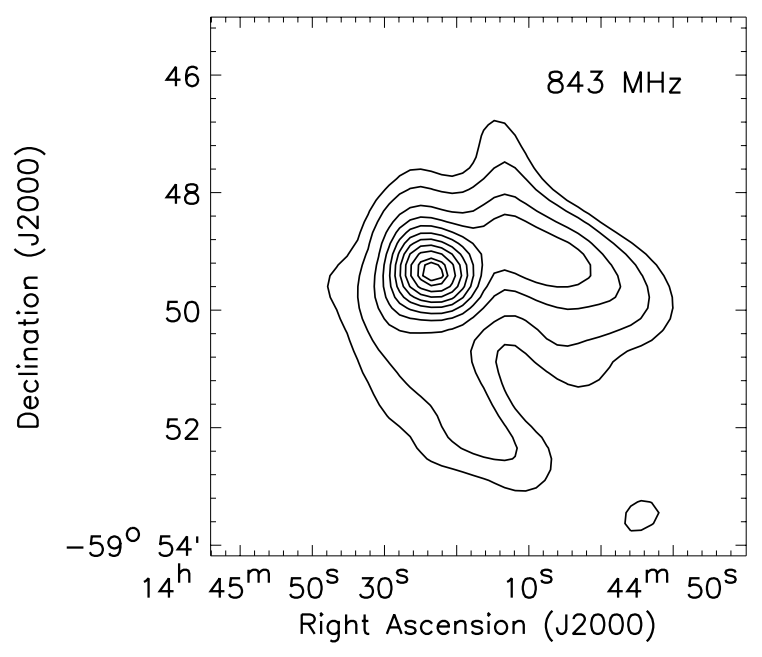

Fig. 5. The SUMSS radio flux density map for the region around IRAS 14416-5937 at $843 \mathrm{MHz}$. The contour levels are at 5, 10, 20, $30,40,50,60,70,80,90$, and $95 \%$ of peak flux of $4.4 \mathrm{Jy} / \mathrm{beam}$. The beam is $\sim 43^{\prime \prime} \times 50^{\prime \prime}$.

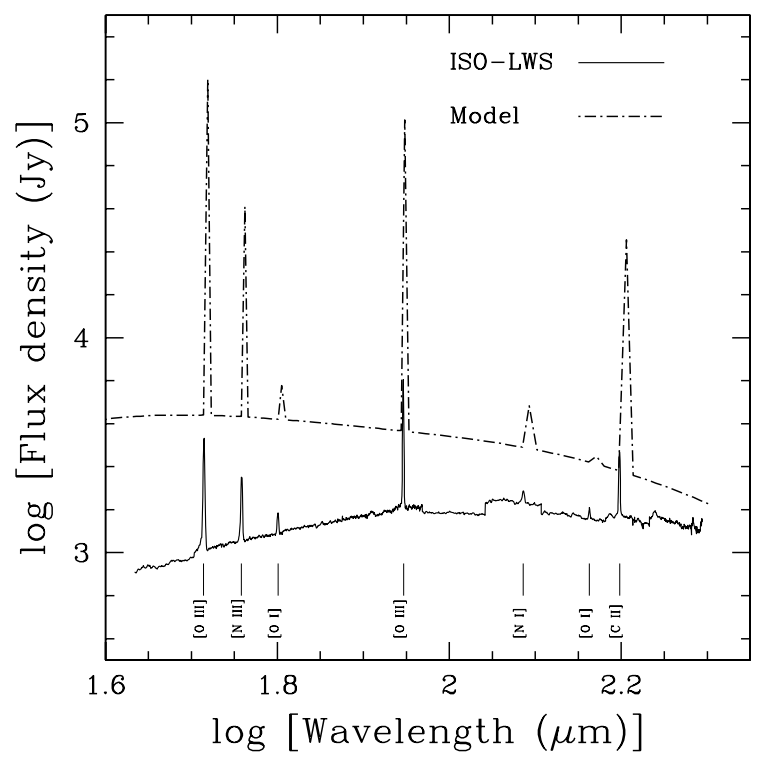

Fig. 6. ISO-LWS spectrum (solid line) shown along with the model calculations (dash-dotted line). The ISO-LWS spectrum is taken for a region $\sim 1.4^{\prime}$ to the north-west of IRAS 14416-5937 - A. However, the model calculations are carried out for IRAS 14416-5937 - A.

removing the underlying local continuum estimated through a polynomial fit to the baseline. The line fluxes, normalised to [C II] line at $158 \mu \mathrm{m}$, are also presented in Table 2 .

\subsection{Embedded cluster}

To study the embedded cluster associated with IRAS 14416-5937, we have selected the 2MASS sources in a square region of size $\sim 8.3^{\prime}$ around the IRAS source. This square region includes the sources $\mathrm{A}$ and $\mathrm{B}$ as well as the dust lane seen in the near- and mid-infrared images (details in Sect. 5.3). A total of 1847 sources are present in the 2MASS catalogue, of which 722 are detected in all $J H K_{\mathrm{s}}$ bands with good quality flags (i.e., rdflg $=1$ to 3 ). The stellar populations of this region have been investigated using the colour-magnitude $(\mathrm{CM} ; J$ vs. $J-H)$ and colour-colour $(\mathrm{CC} ; J-H$ vs. $H-K)$ diagrams of this sample of 722 sources. These are shown
Table 2. Fluxes derived from Gaussian fits to the fine-structure lines observed with ISO-LWS grating positioned at $14^{\mathrm{h}} 45^{\mathrm{m}} 21.0^{\mathrm{s}}-59^{\circ} 48^{\prime} 14^{\prime \prime}$ (J2000).

\begin{tabular}{cccc}
\hline \hline $\begin{array}{c}\text { Element and } \\
\text { ionisation stage }\end{array}$ & $\begin{array}{c}\text { Wavelength } \\
(\mu \mathrm{m})\end{array}$ & $\begin{array}{c}\text { Flux } \\
\left(10^{-17} \mathrm{~W} \mathrm{~cm}^{-2}\right)\end{array}$ & $\begin{array}{c}f \text { (line) } \\
f(\text { (CIII) }\end{array}$ \\
\hline$[\mathrm{C} \mathrm{II}]$ & 157.78 & 1.23 & 1 \\
{$[\mathrm{O} \mathrm{I}]$} & 145.63 & 0.18 & 0.15 \\
{$[\mathrm{~N} \mathrm{II}]$} & 121.84 & 0.36 & 0.29 \\
{$[\mathrm{O} \mathrm{III}]$} & 88.42 & 5.77 & 4.69 \\
{$[\mathrm{O} \mathrm{I}]$} & 63.23 & 0.68 & 0.55 \\
{$[\mathrm{~N} \mathrm{III}]$} & 57.26 & 3.23 & 2.63 \\
{$[\mathrm{O} \mathrm{III}]$} & 51.85 & 8.29 & 6.74 \\
\hline
\end{tabular}

in Fig. 7. In the CM diagram, the ZAMS curves (for a distance of $2.8 \mathrm{kpc}$ ) reddened by $A_{V}=0$ and $20 \mathrm{mag}$, respectively, are presented. The lines tracing the reddening vectors of these main sequence stars are also presented. In the CC diagram, the locii of the main sequence and giant branches are shown. The locus of T Tauri stars (Meyer et al. 1997), the reddening vectors (of main sequence stars, giants and T Tauri stars), and the locus of the Herbig Ae/Be stars (Lada \& Adams 1992) are also shown. We have assumed extinction values of $A_{J} / A_{V}=0.282$, $A_{H} / A_{V}=0.175$, and $A_{K_{\mathrm{s}}} / A_{V}=0.112$ from Rieke \& Lebofsky (1985). All the 2MASS magnitudes as well as the curves are in the Bessel \& Brett (1988) system. In Fig. 7, the sources lying above the reddening curve of the ZAMS spectral type B0 are shown as asterisks, while the stars with infrared excess are shown as open circles. These are sources to the right of the reddening vector drawn from the bottom of the main sequence stars or sources lying in the $\mathrm{T}$ tauri and HeAeBe zones in the $\mathrm{CC}$ diagram. The other sources are represented by dots. It is important to note that the $\mathrm{CM}$ and $\mathrm{CC}$ diagrams are useful tools for estimating the approximate nature of the stellar populations within the cluster in the absence of any spectroscopic data.

While the near-infrared sources in this region have been studied using 2MASS, the near- to mid-infrared sources in this region have been extracted from the Spitzer-GLIMPSE catalogs. In a region identical to the one used for extracting the 2MASS sources, a total of 2087 sources were detected. For our analysis, those sources that have a flux calculation method flag (MF) equal to 0 (good quality) were selected. A total of 130 sources (with $\mathrm{MF}=0$ ) were detected in all the four IRAC bands. All these 130 sources have been plotted in a colour-colour diagram ([3.6]-[4.5] vs. [5.8]-[8.0]), which is shown in Fig. 8. We have used the IRAC colour-colour diagram and the models of Allen et al. (2004) to identify the young stellar objects in this region. The solid-line square in the diagram approximately delineates the region occupied by the Class II sources, whereas the dotted-line square covers the region occupied by the Class I sources, as shown in the models of Allen et al. (2004, see their Fig. 4). There is a region of overlap of these two boxes, and the sources in this region will be referred to as Class I/II sources. In our sample of 130 sources, we find that 14 sources can be classified as Class I, and 11 sources each can be classified as Class I/II, and Class II sources. These are likely to be associated with IRAS 14416-5937 and to be evolving towards the main sequence. In Fig. 8, the open circles denote Class I sources, the open squares represent Class I/II sources, the filled triangles denote Class II sources, and the cross symbols represent the other sources. 

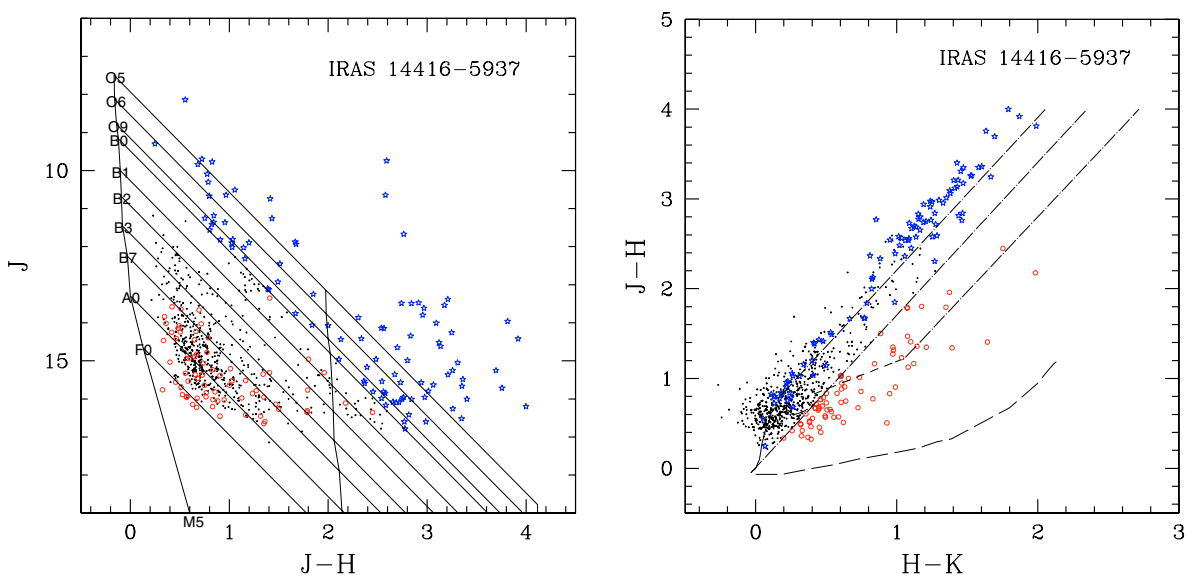

Fig. 7. Colour-magnitude (left) and colour-colour diagram (right) for sources detected in all the three 2 MASS bands for the region around IRAS 14416-5937. In the colour-magnitude diagram, the nearly vertical solid lines from left to right represent the zero age main sequence (ZAMS) curves reddened by $A_{V}=0$, and $20 \mathrm{mag}$, respectively. The slanting lines trace the reddening vectors of these ZAMS stars. In the colour-colour diagram, the locii of the main sequence and giant branches are shown by the solid and dotted lines, respectively. The short-dash line represents the locus of T-Tauri stars. The three parallel dash-dotted straight lines follow the reddening vectors of giants, main sequence stars (or dwarfs), and the T-Tauri stars. The long dashed line represents the locus of Herbig Ae/Be stars. The asterisk symbols represent sources lying above the ZAMS curve B0. The open circles represent sources depicting an infrared excess in the colour-colour diagram. The dots are sources lying below the ZAMS spectral curve of B0 (see text for details).

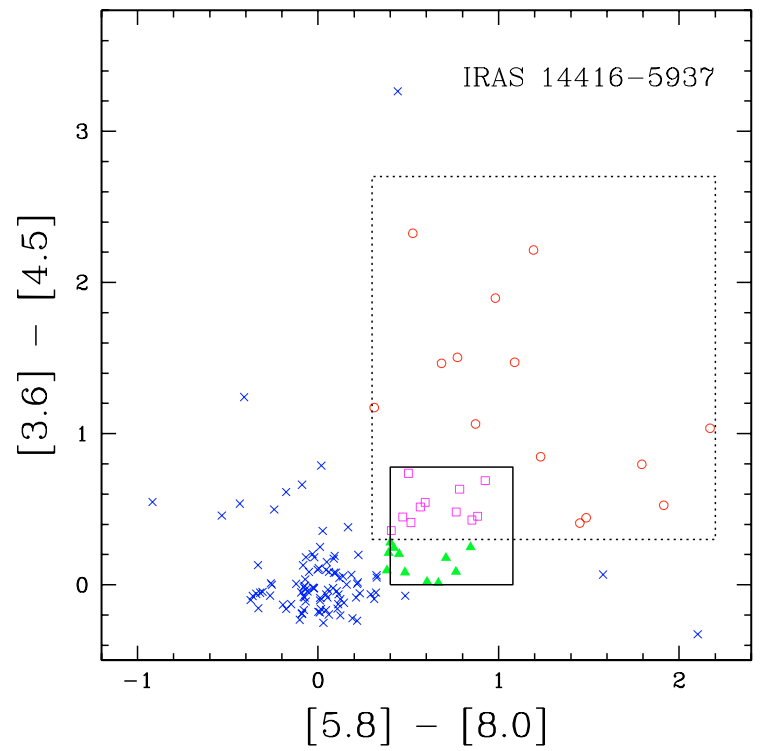

Fig. 8. Colour-colour diagram of the sources detected in all the four bands of Spitzer-IRAC for the region around IRAS 14416-5937. The open circles, open squares, filled triangles, and cross symbols denote Class I, Class I/II, Class II, and other sources, respectively. The solidline square in the diagram approximately delineates the region occupied by the Class II sources, whereas the dotted-line square covers the region occupied by the Class I sources as shown in the models of Allen et al. (2004).

\section{Radiative transfer modelling}

The modelling procedure described in this section has been used to interpret the results obtained.

\subsection{Continuum emission from dust and gas}

We have carried out radiative transfer modelling of the sources IRAS 14416-5937 A and B to obtain the various physical parameters of this region. The radiative transfer equations have been solved assuming a two-point boundary condition for a spherically symmetric cloud of dust and gas. The gas exists throughout the modelled cloud. The dust, however, exists in a spherical shell with a cavity at the centre. The cavity represents the region where dust temperature would be higher than its sublimation temperature due to a stronger radiation field. In the spherical shell where gas and dust co-exist, the gas-to-dust ratio is held constant. The cloud is heated internally by centrally embedded sources and by an external radiation field due to the average Galactic interstellar radiation field (ISRF). The position of the ionisation front depends on the effective temperature and luminosity of the exciting star.

For modelling the observed spectral energy distribution, two types of dust have been explored. The first type of dust grains is from Draine \& Lee (1984), hereafter referred to as DL type of grains. The physical properties of the grains, viz., absorption and scattering efficiencies, the scattering anisotropy factor for all sizes, and the frequencies used in the model were taken from the tables of Draine's homepage ${ }^{1}$, which are computed in a similar manner to that by Laor \& Draine (1993). Three types of the most commonly accepted variety of dust grains have been used here: (i) Graphite; (ii) Astronomical Silicate; and (iii) Silicon Carbide. The second type of dust is from Mezger et al. (1982, hereafter MMP). This type of dust consists of graphite and silicate only, but their absorptive and scattering properties differ substantially from those for the DL case, particularly in the mid-infrared. The relative abundances of the types of grains are used as parameters of our modelling.

The cloud is parameterised by the following quantities: the geometry of the cloud (outer size and inner size of dust cavity), total radial optical depth at a specified wavelength and the radial dust and gas density distribution laws $\left(r^{0}, r^{-1}, r^{-2}\right)$. The gasto-dust ratio is a parameter that is held constant throughout the cloud (except for the central dust-free cavity). The luminosity of the embedded source is obtained by integrating the area under the observed SED. The physical sizes of the cloud, the radial optical depth, dust composition, and the radial density distribution are varied to obtain a good match to the observations. The observed angular sizes and luminosity have been used to constrain

\footnotetext{
${ }^{1}$ http://www . astro.princeton.edu/ draine/dust/
} 

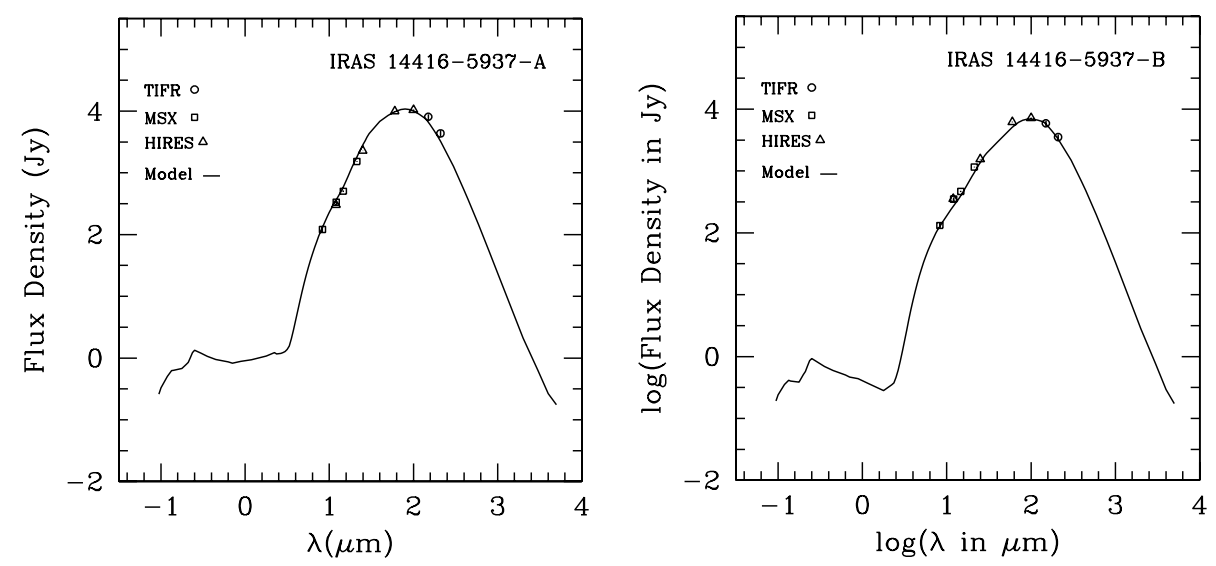

Fig. 9. Comparison of the spectral energy distribution from observations and the best-fit radiative transfer model of IRAS 14416-5937 - A (left) and IRAS 14416-5937 - B (right). The open circles, triangles, and squares represent the TIFR, IRAS-HIRES and MSX data, respectively. The solid line is the best-fit radiative transfer model to the data. See text and Table 3 for details of the model parameters.

Table 3. Best-fit parameters of the radiative transfer models for IRAS 14416-5937 - A and IRAS 14416-5937 - B.

\begin{tabular}{ccccccccc}
\hline \hline $\begin{array}{l}\text { Source } \\
\text { IRAS }\end{array}$ & $\alpha$ & $\begin{array}{c}R_{\max } \\
(\mathrm{pc})\end{array}$ & $\begin{array}{c}R_{\min } \\
(\mathrm{pc})\end{array}$ & $\begin{array}{c}r_{\mathrm{HII}} \\
(\mathrm{pc})\end{array}$ & $\tau_{100}$ & $\begin{array}{c}L \\
\left(10^{5} L_{\odot}\right)\end{array}$ & $\begin{array}{c}\text { Dust } \\
\text { type }\end{array}$ & $\begin{array}{c}M_{\text {dust }} \\
\left(M_{\odot}\right)\end{array}$ \\
\hline $14416-5937-\mathrm{A}$ & 0.0 & 3.2 & 0.025 & 0.5 & 0.007 & 1.3 & $\mathrm{DL}$ & 25 \\
$14416-5937-\mathrm{B}$ & 0.0 & 2.8 & 0.007 & 0.2 & 0.018 & 0.7 & DL & 49 \\
\hline
\end{tabular}

the model. With this scheme, a best-fit model matching the observed SED and angular sizes at selected wavelengths and the radio continuum flux are obtained. Further details of the modelling scheme are given by Mookerjea \& Ghosh (1999).

The SEDs of both these sources (IRAS 14416-5937 A and B) are constructed using the flux densities at the two TIFR bands, the four IRAS bands (from HIRES maps), and the four MSX bands. It may be noted that the position of the peak emission associated with B shifts northward with increasing wavelength. This could be attributed to the cold dust lane. Although the details of the geometry are unclear, we have considered the peak emission of B associated with MSX bands and integrated the flux density in a circle of diameter $3^{\prime}$ around this MSX peak in all the bands. Since A and B are separated by $\sim 2.5^{\prime}$, the flux densities in the slight overlap region are distributed in the ratio of the intensities of $\mathrm{A}$ and $\mathrm{B}$ peaks.

\subsubsection{IRAS 14416-5937 - A}

The total luminosity of this source is $1.4 \times 10^{5} L_{\odot}$ for a distance of $2.8 \mathrm{kpc}$. The best-fit radiative transfer model corresponds to a uniform density distribution of dust and gas. The DL type of dust fits the data better. The relative fraction of the two constituent grain types Si:Gr is 11:89 for the best-fit model. The predicted spectrum by the best-fit model has been compared with the observations in Fig. 9 (left) and the corresponding parameters obtained from this model are tabulated in Table 3. The cloud size (outer radius) is $3.4 \mathrm{pc}$ and the radial optical depth at $100 \mu \mathrm{m}$ is 0.007. A single ZAMS star of spectral type O7-O6.5 has been used as the centrally exciting source. From the model, the radius of the ionised region is determined to be $0.5 \mathrm{pc}$. The radio flux density predicted by the model at $843 \mathrm{MHz}$ is $2.6 \mathrm{Jy}$ for a gas-to-dust ratio of 100 . This is lower than the measured value of 10.2 Jy obtained by integrating within a circular region of radius $0.5 \mathrm{pc}$ around the radio peak (see Fig. 5). This could be due to either gas-to-dust ratio and/or due to clumpy/inhomogeneous medium. Increasing the gas-to-dust ratio, however, does not increase the predicted radio flux beyond $\sim 5 \mathrm{Jy}$. It is, therefore, probable that the difference is due to non-uniform distributions of gas in this region.

\subsubsection{IRAS $14416-5937-B$}

By integrating the observed SED, the total luminosity obtained for IRAS $14416-5937-\mathrm{B}$ is $6.8 \times 10^{4} L_{\odot}$. The best-fit radiative transfer model along with the observed SED is shown in Fig. 9 (right), and the parameters of the best-fit model are presented in Table 3. The best-fit model is a uniform density distribution of gas and dust. The outer size of the cloud is $2.8 \mathrm{pc}$ and the optical depth at $100 \mu \mathrm{m}$ is 0.018 . We have used a single ZAMS star of spectral type O8-O7.5 to carry out the radiative transfer modelling. In the best-fit model, the DL type of dust has been used.

\subsection{Line emission from gas in IRAS 14416-5937 - A}

Since high resolution spectroscopic observations of a region near IRAS 14416-5937 - A are available from ISO-LWS, an attempt has been made to model this source using a sophisticated scheme that includes the gas component with significant details. This scheme, based on CLOUDY, predicts infrared nebular/ionic fine structure line emission from the interstellar gas in IRAS 14416-5937 - A, which has been compared with the ISO-LWS observations.

To model line emission from gas, several prominent elements in the gas phase of the cloud have been considered. Physical processes like thermal balance considering various heating and cooling processes, photoionisation, recombination, collisional excitation and de-excitation, grain photoionisation, and gas-dust coupling have been included in the model. The detailed modelling involves the use of the photoionisation code CLOUDY (Ferland 1996), which has been supplemented with a software scheme developed by Mookerjea \& Ghosh (1999). This scheme improves the modelling by (a) emulating the exact 
Table 4. Emergent line luminosities predicted by the model for IRAS 14416-5937 - A.

\begin{tabular}{|c|c|c|c|}
\hline $\begin{array}{l}\text { Element and } \\
\text { ionisation stage }\end{array}$ & $\begin{array}{r}\text { Wavelength } \\
(\mu \mathrm{m})\end{array}$ & $\begin{array}{r}\text { Luminosity } \\
\qquad\left(L_{\odot}\right)\end{array}$ & $\frac{L(\text { line })}{L([\mathrm{CIII})}$ \\
\hline$[\mathrm{C} \mathrm{II}]$ & 157.78 & 17.91 & 1 \\
\hline$[\mathrm{O} \mathrm{I}]$ & 145.63 & 0.17 & 0.01 \\
\hline$[\mathrm{N} \mathrm{II}]$ & 121.84 & 1.57 & 0.09 \\
\hline [O III] & 88.42 & 121.40 & 6.78 \\
\hline$[\mathrm{O} \mathrm{I}]$ & 63.23 & 2.64 & 0.15 \\
\hline [N III] & 57.26 & 57.70 & 3.22 \\
\hline [O III] & 51.85 & 271.54 & 15.16 \\
\hline$[\mathrm{Ne} \mathrm{III}]$ & 36.04 & 8.20 & 0.46 \\
\hline [Si II] & 34.84 & 6.23 & 0.35 \\
\hline [S III] & 33.50 & 116.71 & 6.52 \\
\hline [O IV] & 25.91 & 1.12 & 0.06 \\
\hline [Ar III] & 21.84 & 2.79 & 0.16 \\
\hline [S III] & 18.69 & 111.75 & 6.24 \\
\hline [Ne III] & 15.57 & 88.77 & 4.96 \\
\hline [Ne II] & 12.82 & 23.81 & 1.33 \\
\hline [S IV] & 10.52 & 50.65 & 2.83 \\
\hline [Ar III] & 8.99 & 35.51 & 1.98 \\
\hline [Ar II] & 7.00 & 2.23 & 0.12 \\
\hline
\end{tabular}

structure of the HII region and (b) including absorption effects of dust (present within the line emitting zones) on the emergent line intensities. Typical HII region abundance of the gas component, tabulated by Ferland (1996) has been taken into consideration. Elements with relative abundance higher than $3.0 \times 10^{-6}$ have been used; these are $\mathrm{H}, \mathrm{He}, \mathrm{C}, \mathrm{N}, \mathrm{O}, \mathrm{Ne}, \mathrm{Mg}, \mathrm{Si}, \mathrm{S}$, and Ar.

The geometry of the cloud has been taken to be identical to that obtained from the modelling of the continuum SED (see Sect. 4.1.1). CLOUDY is run twice. First, it is for the pure gas inner shell. The emerging spectrum comprises of continuum and line emission. This emerging continuum from the inner shell is input to the second shell comprising gas and dust. The line emission from the inner shell in the first run is transported outside through extinction by the dust column in the second shell. The emerging line luminosities from both the shells are finally added to predict the total luminosity. A total of 27 spectral lines in the wavelegth range $2.5-200 \mu \mathrm{m}$ have been considered. The predicted emerging spectrum has been computed by convolving the spectral lines with typical spectral resolutions of ISO-SWS and LWS, viz., 1000 for $2.5 \leq \lambda<12 \mu \mathrm{m}, 20000$ for $12 \leq \lambda<45 \mu \mathrm{m}, 8100$ for $45 \leq \lambda<75 \mu \mathrm{m}$, and 6800 for $75 \leq \lambda<200 \mu \mathrm{m}$.

For IRAS 14416-5937 - A, the emergent spectrum (obtained using the above procedure) shows a total of 18 nebular/ionic lines satisfying our detectability criterion (power in the line $>1 \%$ of power in the neighbouring continuua). The wavelengths and luminosities of these lines are presented in Table 4 . The ratio of luminosity of each line to that of [C II] line at $158 \mu \mathrm{m}$ is also listed. The complete emerging spectrum, including lines from the 10 elements considered as well as the continuum predicted by this model, is shown in Fig. 10.

\section{Discussion}

\subsection{Emission from dust}

The far infrared TIFR (150 and $210 \mu \mathrm{m})$ and IRAS-HIRES (60 and $100 \mu \mathrm{m}$ ) maps probe emission from relatively colder dust in the complex including IRAS 14416-5937 A and B. The temperature of the far infrared emitting cold dust is $\sim 25-30 \mathrm{~K}$ (Fig. 3 ). It is interesting to note that, of the IRAS-HIRES images in the

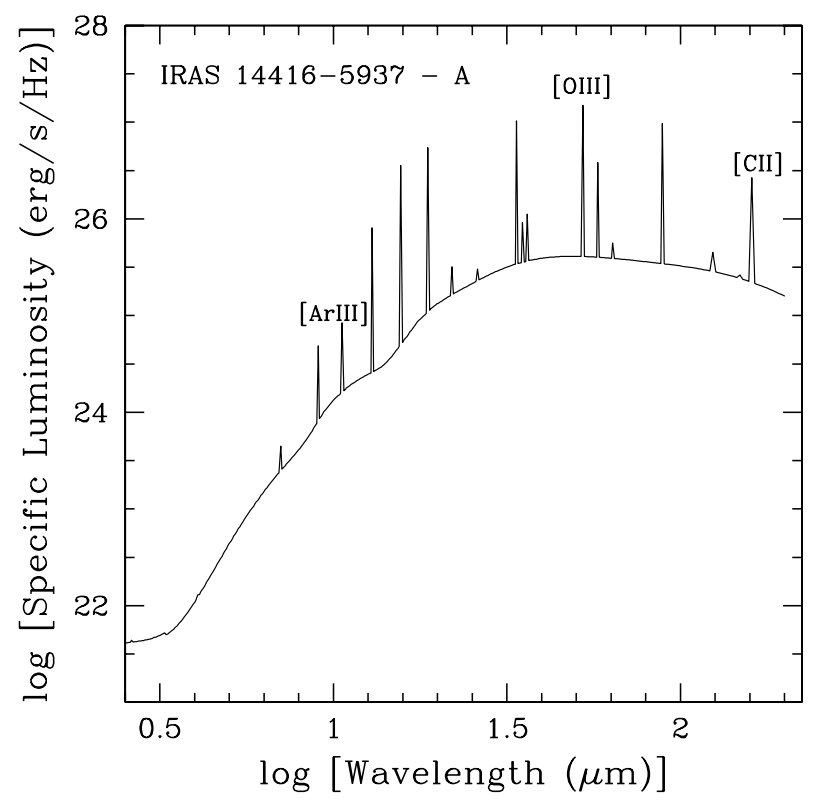

Fig. 10. Emergent spectrum from model calculations of IRAS 144165937 - A. Few lines are identified and the details of the lines are given Table 4.

four wavebands, the peak emission at $12 \mu \mathrm{m}$ is at B, while for the other three bands $(25,60$, and $100 \mu \mathrm{m})$, it is at A. From the IRAS-HIRES maps, too, we see extended dust emission. Also, in all the six maps, there is extended diffuse emission towards the south of source A. The flux densities from the TIFR maps at 150 and $210 \mu \mathrm{m}$ have been used to compute the mass of the dust component using the formulation of Hildebrand (1983) and Sandell (2000). For a temperature of $27 \mathrm{~K}$ and $24 \mathrm{~K}$, obtained for IRAS 14416-5937 A and B (see Fig. 3), we find their dust masses to be 31 and $36 M_{\odot}$, respectively. This is in fairly good agreement with the dust masses of 25 and $49 M_{\odot}$ obtained from the radiative transfer modelling.

From Fig. 3, we see that the observed peak optical depth at $200 \mu \mathrm{m}$ is 0.06 , located near B. However, we find from radiative transfer modelling that the optical depth at $100 \mu \mathrm{m}$ is 0.02 . This apparent inconsistency (of lower value of $\tau_{100}$ compared to $\tau_{200}$ ) could possibly indicate a clumpy/inhomogeneous medium and/or dust grains of different properties than those used here. The modelled UIB emission (Fig. 4) peaks close to A. The UIB emission map shows that both A and B are resolved into two sources each. This is due to higher angular resolution of the MSX maps compared to the IRAS-HIRES and TIFR maps. These secondary peaks could be due to locally higher radiation fields caused by early-type stars (since the UIB is expected to be excited by UV photons). To locate the possible sources responsible for exciting the secondary UIB peaks, we looked at the sources in $2 \mathrm{MASS}$ and Spitzer catalogs. However, we do not find any such candidates around these peaks. This may perhaps be due to the high extinction around IRAS 14416-5937 region.

\subsection{Emission from gas}

We compare radio continuum emission from the SUMSS radio map at $843 \mathrm{MHz}$ with the high angular resolution map at $6.67 \mathrm{GHz}$ of Walsh et al. (1998). The high angular resolution $\left(\sim 1.5^{\prime \prime}\right)$ map at $6.67 \mathrm{GHz}$ shows two main peaks at $\left(\alpha_{2000}, \delta_{2000}\right)=\left(14^{\mathrm{h}} 45^{\mathrm{m}} 22.54^{\mathrm{s}},-59^{\circ} 49^{\prime} 37.3^{\prime \prime}\right)$ and 
$\left(\alpha_{2000}, \delta_{2000}\right)=\left(14^{\mathrm{h}} 45^{\mathrm{m}} 22.25^{\mathrm{s}},-59^{\circ} 49^{\prime} 39.5^{\prime \prime}\right)$. These are nearly $14^{\prime \prime}$ and $17^{\prime \prime}$ offset from the SUMSS peak at $843 \mathrm{GHz}$, respectively. This could be either due to the effect of lower angular resolution of the SUMSS map or the effect of opacity. It is also important to note that the radio map at $6.67 \mathrm{GHz}$ at high angular resolution covers only the very compact features in this HII region. A comparison of the radio peaks with the peaks of UIB emission shows that the secondary peak near A of the UIB emission is close $\left(\sim 12^{\prime \prime}\right)$ to the high angular resolution radio peaks at $6.67 \mathrm{GHz}$ of Walsh et al. (1998).

In the ISO-LWS spectrum, we notice that the cooling lines from the Photo-Dissociated Regions (PDRs): [O I] 63, $145 \mu \mathrm{m}$ and [C II] $158 \mu \mathrm{m}$ have been clearly detected. In addition, lines coming from the higher excitation potential ions such as [N II] $122 \mu \mathrm{m}$, [N III] $57 \mu \mathrm{m}$, as well as [O III] 52 and $88 \mu \mathrm{m}$ lines are also detected. The maximum flux is observed in the [O III] $52 \mu \mathrm{m}$ line. Since the spectrum is taken at a position that is located around $\sim 1.4^{\prime}$ to the north-west of IRAS 14416-5937-A, it is likely that the observed fluxes in the highly ionised species like [O III] and [N III] are due to an extended component of low density ionised gas rather than compact source(s). In the highest density regions of compact cores, these lines are collisionally deexcited (Morisset et al. 2002). The ratio of fluxes in [O III] lines has been used to estimate the electron density, $n_{\mathrm{e}} \sim 300 \mathrm{~cm}^{-3}$ in this region (details are given in Appendix A). Using the fluxes in [N III] $57 \mu \mathrm{m}$ and [N II] $122 \mu \mathrm{m}$ lines, the effective temperature of the ionising radiation is found to be $\sim 37500 \mathrm{~K}$ (details in Appendix A). This compares well with the effective temperature, $39500 \mathrm{~K}$, of the centrally exciting source required by the radiative transfer model (see Sect. 4.1.1). Next, we consider the flux ratio of [N III] $57 \mu \mathrm{m}$ to [O III] $52 \mu \mathrm{m}$. Since the ionisation potentials of these two ions are similar $\left(\mathrm{O}^{++}=35.1 \mathrm{eV}\right.$; $\left.\mathrm{N}^{++}=29.6 \mathrm{eV}\right)$, they are likely to sample the same volume of gas (Mizutani et al. 2002). In our case, the intensity ratio from the observed ISO-LWS spectrum is $I([\mathrm{~N} \mathrm{III]}] 57) / I([\mathrm{OIII}] 52) \sim$ 0.40 . Mizutani et al. (2002) find that this ratio is almost constant and obtain the value of $\sim 0.3$ for the optically bright regions of the Carina nebula and a value ranging from $0.25-0.5$ for the surrounding region. We have also estimated the density and the radiation field using the ratio $\left([\mathrm{CII}]+[\mathrm{OI}]^{63}\right) / L_{\mathrm{FIR}}$, which is a measure of the gas heating efficiency and the ratio of line intensities $[\mathrm{C} \mathrm{II}] /[\mathrm{OI}]^{63}$. The total far infrared emission is obtained by integrating the ISO-LWS continuum. We obtain FIR flux for this region to be $7.1 \times 10^{-11} \mathrm{~W} \mathrm{~m}^{-2}$. The gas density and radiation field are found to be $\sim 100 \mathrm{~cm}^{-3}$ and $\sim 300 G_{0}\left(G_{0}\right.$ is Habing Field $=1.6 \times 10^{-6} \mathrm{~W} \mathrm{~m}^{-2}$ ), respectively, from the Fig. 4 (right) of Peeters et al. (2005).

Figure 6 shows comparison of the spectra from ISO-LWS and the model calculations. The flux densities from the model calculations are higher than that observed from ISO. This is expected since the ISO-LWS beam is centred $1.4^{\prime}$ away from the IRAS 14416-5937 - A peak. Also, the ISO-LWS beam is of size $84^{\prime \prime}$, whereas the model computes the total emergent intensities from the entire cloud. It is instructive to compare the observed and modelled fine structure line ratios, normalised with respect to [C II] $158 \mu \mathrm{m}$ line. The ratio of the lines corresponding to the doubly ionised atoms [O III] 52 and $88 \mu \mathrm{m}$ and [N III] $57 \mu \mathrm{m}$ are overestimated by the model as compared to the observations by a factor of up to 2 . On the other hand, the ratio of the lines [O I] $63 \mu \mathrm{m}$ and [N II] $122 \mu \mathrm{m}$ is underestimated by the model by a factor of $\sim 3$. The major difference is found for the [O I] $145 \mu \mathrm{m}$ line ratio, which is underestimated by the model by a factor of $\sim 16$.

\subsection{Association with the cluster}

Next we consider the embedded star clusters associated with the IRAS 14416-5937 region based on the near- and mid-infrared emission. From the 2MASS CM diagram in Fig. 7 (left), we find that there are 98 sources lying above the reddening curve of the ZAMS spectral type B0 for a distance of $2.8 \mathrm{kpc}$. It is unlikely that all these objects are ZAMS stars of spectral type earlier than B0 and associated with the cluster since the combined luminosity of these stars would be much higher than the observed luminosity. It is reasonable to consider that many of these objects are foreground stars or bright background giants not associated with the star-forming region, although a few of these may be O-B stars belonging to the cluster. From the 2MASS CC diagram, in Fig. 7 (right), we find that there are 72 sources having an infrared excess (based on the CC diagram). These sources have been overplotted on the 2MASS $K_{\mathrm{s}}$ band image of the region around IRAS 14416-5937 in Fig. 11. The grayscale 2MASS image of this region shows diffuse emission apart from a number of sources. A dust lane $\sim 3.5 \mathrm{pc}$ long running diagonally across the image commencing from the source A is clearly observed. As compared to the rest of the image where a number of 2MASS sources are detected, very few sources are seen in the region of the dust lane. From the spatial distribution of the sources in the figure, we find that most of the 2MASS sources within the dust lane are those with infrared excess. A larger number of sources with infrared excess are present in the IRAS 14416-5937 - A region as compared to $\mathrm{B}$. On the other hand, we find sources of "spectral type" earlier than B0 clustered near IRAS 144165937 - B. This suggests the possibility that the complex B could be more evolved than A. While the sources lying above the reddening vector of ZAMS spectral type B0 in the 2MASS CM diagram are distributed everywhere in the image other than the dust lane, we see a larger number of sources with infrared excess below the dust lane (i.e., bottom right part of the image). These objects with infrared excess are likely to be pre-main sequence candidate objects in this star-forming region. We have also compared the statistics of the infrared excess sources with that obtained from two neighbouring control fields of the same size. We find that the number of infrared excess sources in the star-forming field is larger than those obtained from the control fields by a factor of $\sim 2$.

In Fig. 11, the SUMSS radio contours at $843 \mathrm{MHz}$ have been overplotted on the grayscale $K_{\mathrm{s}}$ band image of 2MASS. Within the radio nebulosity, we find that there are a number of asterisks (sources lying above the reddening vector of ZAMS spectral type B0 and without infrared excess in the 2MASS CM diagram). Within the $30 \%$ contour level, there are six asterisks. The details of these sources are listed in Table 5. Among these, J14452143-5949251 is closest to the radio peak $\left(\sim 15.7^{\prime \prime}\right)$. We, however, do not find a Spitzer-GLIMPSE counterpart of J14452143-5949251 within a search radius of $0.8^{\prime \prime}$ (search radius used for associating the 2MASS and SpitzerGLIMPSE sources). An investigation of the IRAC images reveals strong emission at the location of this source in all the $4 \operatorname{IRAC}(3.6,4.5,5.8$, and $8.0 \mu \mathrm{m})$ bands. The spectral energy distribution of this source is shown in Fig. 12. The fluxes in the IRAC bands were obtained by integrating within an aperture of diameter $6^{\prime \prime}$ (and applying the aperture corrections) centred on peak emission at $3.6 \mu \mathrm{m}$ after subtracting the background. From the SED, we observe that this is a young reddened source possibly with a dust envelope/disk around it. The lower limit of the luminosity of this source obtained after integrating the SED is $\sim 50 L_{\odot}$. It would be interesting to do spectroscopy of 
Table 5. Details of 2MASS PSC sources lying above the reddening vector of the ZAMS spectral type O6 in the 2MASS ( $J-H$ vs. $J$ ) CM diagram and lying within the $30 \%$ contour of the radio peak at $843 \mathrm{MHz}$ (see Fig. 11 and text for details).

\begin{tabular}{cccccc}
\hline \hline $\begin{array}{c}\text { 2MASS PSC } \\
\text { designation }\end{array}$ & $\begin{array}{c}\alpha_{2000} \\
(\mathrm{deg})\end{array}$ & $\begin{array}{c}\delta_{2000} \\
(\mathrm{deg})\end{array}$ & $\begin{array}{c}J \\
(\mathrm{mag})\end{array}$ & $\begin{array}{c}\mathrm{H} \\
(\mathrm{mag})\end{array}$ & $\begin{array}{c}K_{\mathrm{s}} \\
(\mathrm{mag})\end{array}$ \\
\hline $\mathrm{J} 14450407-5948596$ & 221.266960 & -59.816566 & $11.17 \pm 0.02$ & $9.80 \pm 0.02$ & $9.33 \pm 0.03$ \\
$\mathrm{~J} 14450584-5949317$ & 221.274341 & -59.825478 & $11.83 \pm 0.02$ & $10.22 \pm 0.02$ & $9.44 \pm 0.02$ \\
$\mathrm{~J} 14451702-5949336$ & 221.320956 & -59.826023 & $13.40 \pm 0.03$ & $10.31 \pm 0.03$ & $8.84 \pm 0.03$ \\
$\mathrm{~J} 14451788-5949360$ & 221.324514 & -59.826691 & $15.88 \pm 0.19$ & $13.11 \pm 0.08$ & $11.66 \pm 0.08$ \\
$\mathrm{~J} 14452143-5949251$ & 221.339322 & -59.823666 & $14.12 \pm 0.04$ & $10.95 \pm 0.03$ & $9.30 \pm 0.02$ \\
$\mathrm{~J} 14452450-5950084$ & 221.352090 & -59.835682 & $14.26 \pm 0.04$ & $10.43 \pm 0.02$ & $8.58 \pm 0.02$ \\
\hline
\end{tabular}

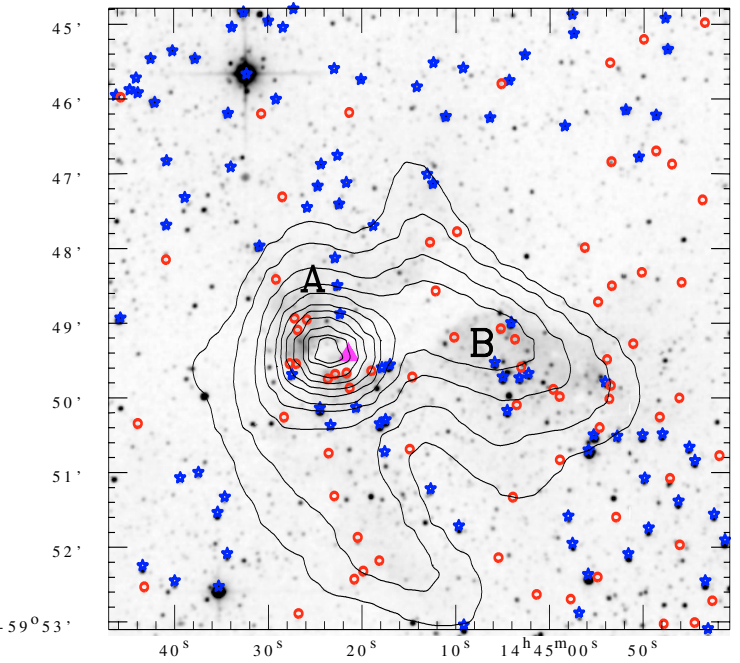

Fig. 11. The radio contours (SUMSS; $843 \mathrm{MHz}$ ) overlaid over the 2MASS $K_{\mathrm{s}}$ band image for the region around IRAS 14416-5937. The labelled axes are in J2000 coordinates. The regions "A" and "B" are shown and various near-infrared sources are marked. The asterisk symbols represent sources of spectral type earlier than B0, and the circles denote the infrared excess sources. The solid triangle represents J14452143-5949251 (2MASS source closest to radio peak detected in all the three $\left(J H K_{\mathrm{s}}\right)$ bands and of spectral type earlier than $\mathrm{O} 6$ from the CM diagram).

this source, J14452143-5949251, to ascertain its spectral type and additional details.

We have attempted to identify protostars and pre mainsequence objects among the Spitzer-GLIMPSE sources located around IRAS 14416-5937, detected in all the four IRAC bands, based on the models of Allen et al. (2004). These sources have been overplotted on the Spitzer-GLIMPSE $5.8 \mu \mathrm{m}$ band image, shown in Fig. 13. The details of these young Spitzer objects are available in Table A.6 (available only as electronic table). We have also searched for 2MASS counterparts of these young GLIMPSE objects in this star-forming region. A search radius of $0.8^{\prime \prime}$ has been taken as a criterion for associating the 2MASS and Spitzer-GLIMPSE objects. While only 2 of the 14 Class I objects have 2MASS counterparts, 10 out of the 11 Class I/II objects have 2MASS counterparts and all the 11 Class II objects have 2MASS counterparts. This is probably because most Class I objects suffer large amounts of extinction. We also searched for Spitzer-GLIMPSE counterparts to the 2MASS infrared excess objects detected in all the bands of 2MASS $\left(J H K_{\mathrm{s}}\right)$. We find that 36 out of 72 2MASS IR excess objects have Spitzer-GLIMPSE counterparts. It is to be noted that most of these objects are towards the lower right part of the image with few along the farther end of the dust lane. The details of these 2MASS IR excess objects with Spitzer GLIMPSE

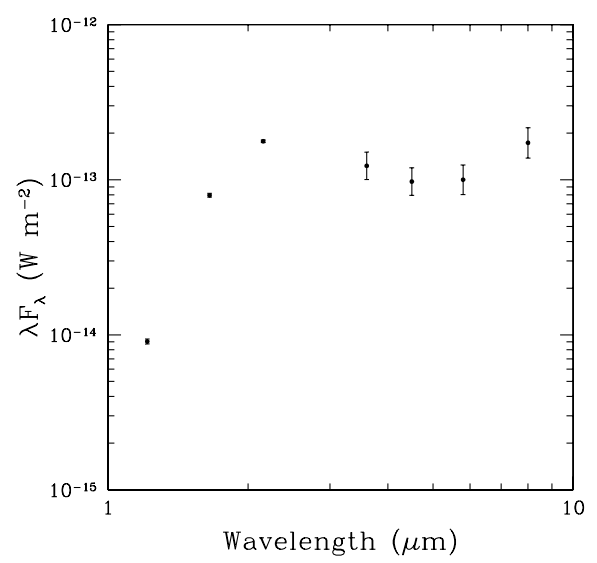

Fig. 12. The spectral energy distribution of J14452143-5949251 (2MASS source closest to radio peak detected in all the three $\left(J H K_{\mathrm{s}}\right)$ bands and of spectral type earlier than $\mathrm{O} 6$ from the CM diagram) constructed using the fluxes from 2MASS and Spitzer-GLIMPSE (details in text).

counterparts are given in Table A.7 (available only as electronic table).

The presence of diffuse near-infrared emission around IRAS 14416-5937 - A and B in Figs. 11 and 13 indicates that these are relatively evolved regions. The presence of HII regions around them is seen from the radio image in Fig. 5. The $\mathrm{OH}$ (Caswell \& Haynes 1987), $\mathrm{H}_{2} \mathrm{O}$ (Caswell et al. 1989), and $\mathrm{CH}_{3} \mathrm{OH}$ (Walsh et al. 1998) masers are marked in the figure in different colours. All these three masers are located close to $\mathrm{A}$, near the peak of radio emission. The $\mathrm{H}_{2} \mathrm{O}$ maser is closest $\left(\sim 12^{\prime \prime}\right)$ to the radio peak. The dust lane is very clearly seen in the IRAC images. It is interesting to note that the dust lane is lined up with only Class I sources. The dust lane has Class I sources extending up to $\sim 5^{\prime}$. It is to be further noted that Class I and Class I/II, as well as Class II sources are present further along the dust lane and beyond B. Below B (i.e., the bottom right part of the image), we find Class I/II and Class II sources. As seen earlier, most of the 2MASS infrared excess sources with Spitzer-GLIMPSE counterparts are also located here. It is likely that the sources here represent an earlier generation of stars in the sequence of star formation. The other sources are distributed over the entire image, but away from the dust lane.

From this distribution, the following scenario emerges: it is evident that star formation activity is in progress along the dust lane. Hence the deeply embedded Class I sources are seen along the dust lane. This suggests a possibility of star formation being triggered by the expanding HII region. A radio map at high angular resolution with diffuse features would help in confirming this. Further along and below the dust lane, the emergence of Class II sources indicates an advanced stage of star formation here. It is not clear what could have caused the star formation 


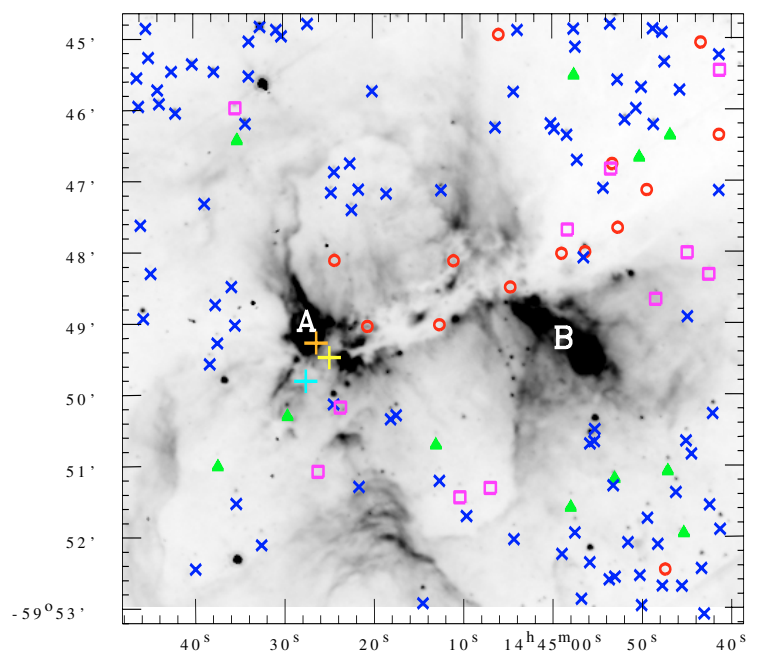

Fig. 13. Spitzer-GLIMPSE sources detected in all the four IRAC bands overlaid over the Spitzer-GLIMPSE $5.8 \mu \mathrm{m}$ band image for the region around IRAS 14416-5937. The labelled axes are in J2000 coordinates. The open circles, open squares, filled triangles, and cross symbols represent the Class I, Class I/II, Class II, and the other sources, respectively (see text for details). The sources "A" and "B" are marked. The $\mathrm{OH}$, $\mathrm{H}_{2} \mathrm{O}$, and $\mathrm{CH}_{3} \mathrm{OH}$ maser positions are shown by the light blue, yellow, and orange plus symbols, respectively.

further along the dust lane. It would be very interesting to map this region in molecular lines, which trace high density like CS, etc. Imaging in the molecular near-infrared line of $\mathrm{H}_{2}$ would help in detecting shocked gas, if any, along the edges of the lane.

\section{Summary}

The massive star-forming region associated with IRAS 144165937 has been studied using the (near, mid-, and far) infrared wavebands. The dust and gas environment as well as the stellar sources of this region have been probed using data from the TIFR balloon-borne telescope, MSX, IRAS-HIRES, SpitzerGLIMPSE, 2MASS, and ISO. The spatial distribution of far infrared emission from cold dust at 150 and $210 \mu \mathrm{m}$ has been obtained along with the maps of optical depth $\left(\tau_{200}\right)$ and colour temperature, $T(150 / 210)$. Using MSX data, the emission from warm dust and UIBs in this region has been studied. This region comprises two sources: A (east) and B (west), as well as a dust lane due north-west of A. Using 2MASS PSC as well as the GLIMPSE catalogs, the near- and mid-infrared sources associated with this region have been studied using colour-magnitude and colour-colour diagrams. The atomic fine structure lines from the ISO-LWS spectrum of a region close to A have been used in estimating the electron density $\left(n_{\mathrm{e}} \sim 300 \mathrm{~cm}^{-3}\right)$, as well as the effective temperature of the ionising radiation $(37500 \mathrm{~K})$ in this region. Self-consistent radiative transfer modelling constrained by observations has been carried out through spherical gas-dust clouds for both the sources (A and B). A constant radial density distribution $\left(n(r) \propto r^{0}\right)$ is preferred. The geometric details of the gas-dust clouds, the dust composition and optical depths, etc. have been obtained for the best-fit models. We have also carried out the modelling of line emission from source A using CLOUDY. The line ratios obtained from the model have been compared with the ISO-LWS spectrum of the region close to A. The Spitzer-GLIMPSE sources detected in all the IRAC bands (3.6, 4.5, 5.8, $8.0 \mu \mathrm{m}$ ) have been classified into Class I (14), Class I/II (11), and Class II (11) sources based on the models of
Allen et al. (2004). Their spatial distribution shows that Class I sources line up the dust lane. The Class II sources are found further along the dust lane indicating this to be a more evolved region. This suggests that the star formation is occuring along the dust lane possibly triggered by the shocks of the expanding $\mathrm{HII}$ regions of $\mathrm{A}$ and $\mathrm{B}$.

Acknowledgements. We thank the anonymous referee for useful suggestions that improved the paper. It is a pleasure to thank the members of the Infrared Astronomy Group at TIFR for their support during laboratory tests and balloon flight campaigns. All members of the Balloon Group and Control Instrumentation Group of the TIFR Balloon Facility, Hyderabad, are thanked for their technical support during the flight. We thank M. Walmsley and L. Testi for many useful suggestions regarding the improvement of this work. We thank A. Walsh for providing us the radio continuum map of IRAS 14416-5937 at $6.67 \mathrm{GHz}$

We thank IPAC, Caltech, for providing us the HIRES-processed IRAS products. This research made use of data products from the Midcourse Space Experiment Processing of the data was funded by the Ballistic Missile Defense Organization with additional support from NASA Office of Space Science. This research has also made use of the NASA/ IPAC Infrared Science Archive, which is operated by the Jet Propulsion Laboratory, Caltech, under contract with the NASA. This publication makes use of data products from the Two Micron All Sky Survey, which is a joint project of the University of Massachusetts and the Infrared Processing and Analysis Center/California Institute of Technology, funded by the NASA and the NSF. This work is based in part on observations made with the Spitzer Space Telescope, which is operated by the Jet Propulsion Laboratory. The MOST is operated by the University of Sydney and supported in parts by grants from the Australian Research Council. Based on observations with ISO, an ESA project with instruments funded by ESA Member States (especially the PI countries: France, Germany, the Netherlands, and the UK) and with the participation of ISAS and NASA.

\section{Appendix A: Electron density and effective temperature of ionising radiation from ISO LWS spectrum}

The ratio of flux in [O III] $52 \mu \mathrm{m}$ to that in [O III] $88 \mu \mathrm{m}$ (hereafter denoted as [O III] 52/88) can be used to estimate the electron density, $n_{\mathrm{e}}$, in this region (Rubin et al. 1994). This is because these are atomic fine structure lines of the same ionic species and are emitted from levels with nearly the same excitation temperature. The ratio [O III] 52/88 1.4 (see Table 2) implies $n_{\mathrm{e}} \sim 300 \mathrm{~cm}^{-3}$ from the semi-empirical treatment of Rubin et al. (1994).

It is also possible to estimate the effective temperature of the ionising radiation $\left(T_{\text {eff }}\right)$ using the fluxes in the [N III] $57 \mu \mathrm{m}$ and [N II] $122 \mu \mathrm{m}$ lines under the assumption that the nebula is ionisation bounded (Rubin et al. 1994). The volume emissivities of [N III] $57 \mu \mathrm{m}$ and [N II] $122 \mu \mathrm{m}$ were obtained for $n_{\mathrm{e}}$ determined above. It is possible to derive the ion abundance ratio $\mathrm{N}^{++} / \mathrm{N}^{+}$using these volume emissivities and fluxes in the lines (Fig. 4 of Rubin et al. 1994). This abundance ratio is found to be $\mathrm{N}^{++} / \mathrm{N}^{+}=0.91$, which corresponds to an effective temperature of $\sim 37500 \mathrm{~K}$.

\section{References}

Allen, L. E., Calvet, N., D'Alessio, P., et al. 2004, ApJS, 154, 363 Aumann, H. H., Fowler, J. W., \& Melnyk, M. 1990, AJ, 99, 1674

Benjamin, R. A., Churchwell, E., Babler, B. L., et al. 2003, PASP, 115, 953 Bessel, M. S., \& Brett, J. M. 1988, PASP, 100, 1134

Bock, D. C., Large, M. I., \& Sadler, E. M. 1999, AJ, 117, 1578

Bronfman, L., Nyman, L.-A., \& May, J. 1996, A\&AS, 115, 81

Busfield, A. L., Purcell, C. R., Hoare, M. G., et al. 2006, MNRAS, 366, 1096

Caswell, J. L., \& Haynes, R. F. 1987, AuJPh, 40, 215

Caswell, J. L., Batchelor, R. A., Forster, J. R., \& Wellington, K. J. 1989, AuJPh, 42,331

Caswell, J. L., Vaile, R. A., Ellingsen, S. P., Whiteoak, J. B., \& Norris, R. P. 1995, MNRAS, 272, 96 
Cernicharo, J. 2004, ApJ, 600, 214

Clegg, P. E., Ade, P. A. R., Armund, C., et al. 1996, A\&A, 315, L38

Draine, B. T., \& Lee, H. M. 1984, ApJ, 285, 89

Fazio, G. G., Hora, J. L., Allen, L. E., et al. 2004, ApJS, 154, 10

Ferland, G. J. 1996, Hazy, a brief introduction to CLOUDY, Univ. of Kentucky, Dept. of Phys. and Astron. Internal Reports

Gardner, F. F., \& Whiteoak, J. B. 1984, MNRAS, 210, 23

Ghosh, S. K., Iyengar, K. V. K., Rengarajan, T. N., et al. 1988, ApJ, 330, 928

Ghosh, S. K., \& Ojha, D. K. 2002, A\&A, 288, 326

Gull, S. F., \& Daniell, G. J. 1978, Nature, 272, 686

Hildebrand, R. H. 1983, QJRAS, 24, 267

Huang, M., Bania, T. M., Bolatto, A., et al. 1999, ApJ, 517, 282

Juvela, M. 1996, A\&AS, 118, 191

Lada, C. J., \& Adams, F. C. 1992, ApJ, 393, 278

Laor, A., \& Draine, B. T. 1993, ApJ, 402, 441

Lloyd, C., Lerate, M. R., \& Grundy, T. W. 2003, Uniformly Processed LWS L01 Spectra, ISO Technical Note 17 (Madrid: ISO Data Centre)

Meyer, M. R., Calvet, N., \& Hillenbrand, L. A. 1997, AJ, 114, 288

Mezger, P. G., Mathis, J. S., \& Panagia, N. 1982, A\&A, 105, 372
Mizutani, M., Onaka, T., \& Shibai, H. 2002, A\&A, 382, 610

Mookerjea, B., \& Ghosh, S. K. 1999, J. Astrophys. Astr., 20, 1

Mookerjea, B., Ghosh, S. K., Rengarajan, T. N., Tandon, S. N., \& Verma, R. P. 2000, AJ, 120, 1954

Morisset, C., Schaerer, D., Martín-Hernández, N. L., et al. 2002, A\&A, 386, 558 Peeters, E., Martín-Hernández, N. L., Rodríguez-Fernández, N. J., \& Tielens, X. 2005, Space Sci. Rev. ISO Special Issue (Springer)

Price, S. D., Egan, M. P., Carey, S. J., Mizuno, D. R., \& Kuchar, T. A. 2001, AJ, 121,2819

Rieke, G. H., \& Lebofsky, M. J. 1985, ApJ, 288, 618

Rubin, R. H., Simpson, J. P., Lord, S. D., et al. 1994, ApJ, 420, 772

Sandell, G. 2000, A\&A, 358, 242

Scoville, N. Z., \& Kwan, J. 1976, ApJ, 206, 718

Vilas-Boas, J. W. S., \& Abraham, Z. 2000, A\&A, 355, 1115

Walsh, A. J., Burton, M. G., Hyland, A. R., \& Robinson, G. 1998, MNRAS, 301, 640

Werner, M. W., Roellig, T. L., Low, F. J., et al. 2004, ApJS, 154, 1

White, G. J., \& Phillips, J. P. 1983, MNRAS, 202, 255

Whiteoak, J. B., Otrupcek, R. E., \& Rennie, C. J. 1982, PASAu, 4, 434 
S. Vig et al.: Infrared study of IRAS 14416-5937, Online Material $p 1$

\section{Online Material}




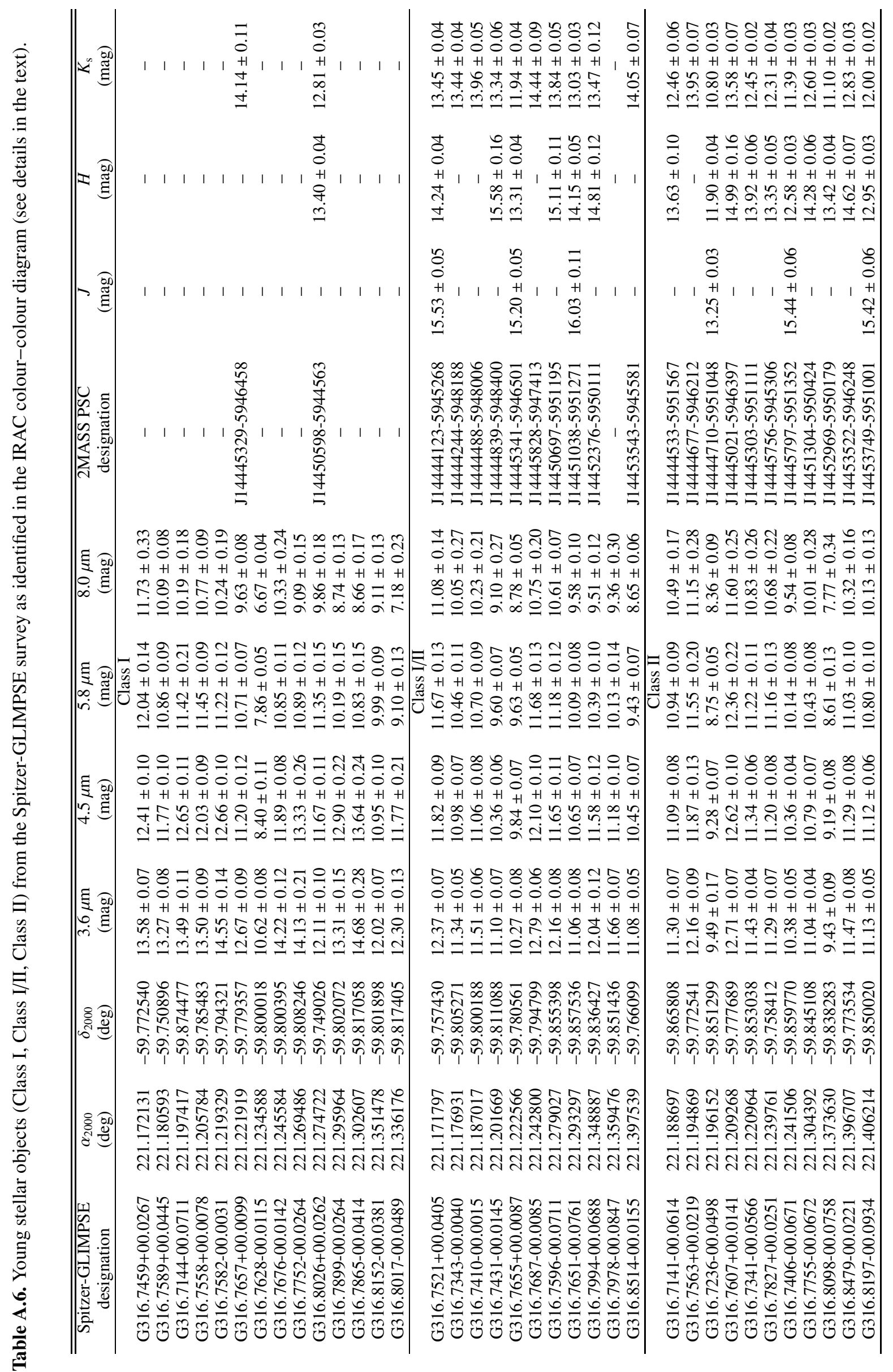




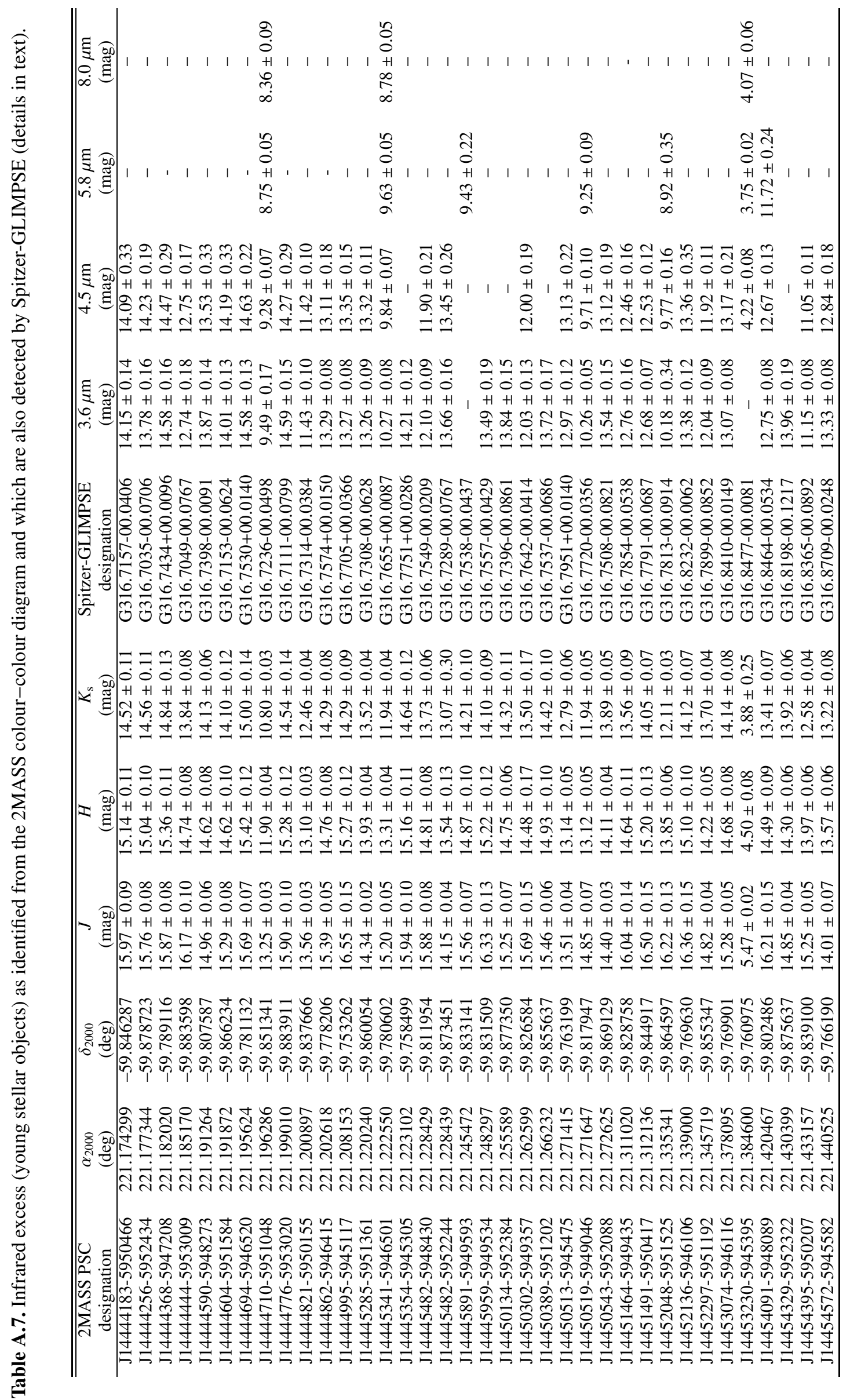

